\title{
Running Your Best Triathlon Race
}

\author{
Naroa Etxebarria, Jackson Wright, Hamish Jeacocke, Cristian Mesquida, and David B. Pyne
}

\begin{abstract}
Negative or evenly paced racing strategies often lead to more favorable performance outcomes for endurance athletes. However, casual inspection of race split times and observational studies both indicate that elite triathletes competing in Olympic-distance triathlon typically implement a positive pacing strategy during the last of the 3 disciplines, the $10-\mathrm{km}$ run. To address this apparent contradiction, the authors examined data from 14 International Triathlon Union elite races over 3 consecutive years involving a total of 725 male athletes. Analyses of race results confirm that triathletes typically implement a positive running pace strategy, running the first lap of the standard 4-lap circuit substantially faster than laps $2(\sim 7 \%), 3(\sim 9 \%)$, and $4(\sim 12 \%)$. Interestingly, mean running pace in lap 1 had a substantially lower correlation with 10-km run time $(r=.82)$ than both laps 2 and 3. Overall triathlon race performance (ranking) was best associated with run performance $(r=.82)$ compared with the swim and cycle sections. Lower variability in race pace during the $10-\mathrm{km}$ run was also reflective of more successful run times. Given that overall race outcome is mainly explained by the $10-\mathrm{km}$ run performance, with top run performances associated with a more evenly paced strategy, triathletes (and their coaches) should reevaluate their pacing strategy during the run section.
\end{abstract}

Keywords: endurance performance, individualized racing, Olympic distance, pacing strategy

Olympic-distance triathlon comprises a sequential $1.5-\mathrm{km}$ swim, 40-km cycle, and 10-km run. Although the ability to perform the 3 disciplines at a high level is critical for competitive success, ${ }^{1,2}$ it appears the run section is the main determinant in Olympicdistance triathlon. ${ }^{2-4}$ The last of the 3 disciplines, the run section, represents a relatively small portion of the overall triathlon race ( $\sim 25 \%$ by duration), and despite anecdotal evidence that it has the largest influence on overall race outcome in the recent years, the question is mostly unanswered. Several factors influence running performance in triathlon, such as performance in the previous swimming and cycling sections, especially the variability in cycling power output, ${ }^{5}$ and also pacing during the run. ${ }^{6}$ Given the rapid evolution in triathlon in the last decade, early studies on pacing in triathlon pacing might be outdated, and the supporting evidence was either single-race based ${ }^{3,4,7}$ or from a simulated race in the laboratory, ${ }^{6}$ which limits their application to contemporary triathlon. Single event-based evidence also shows an inverse correlation between variability in running pace and overall simulated $10-\mathrm{km}$ triathlon run time. ${ }^{7}$ Apart from single-race outcomes or simulated race conditions, it is unclear what pacing strategies elite triathletes adopt in modern Olympic-distance triathlon.

(C) 2021 The Authors. Published by Human Kinetics, Inc. This is an Open Access article distributed under the terms of the Creative Commons Attribution-NonCommercial-NoDerivatives 4.0 International License, CC BY-NC-ND 4.0, which permits the copy and redistribution in any medium or format, provided it is not used for commercial purposes, no modifications are made, appropriate credit is given, and a link to the license is provided. See http://creativecommons.org/licenses/ by-nc-nd/4.0. This license does not cover any third-party material that may appear with permission in the article. For commercial use, permission should be requested from Human Kinetics, Inc., through the Copyright Clearance Center (http://www. copyright.com).

Etxebarria, Wright, and Pyne are with the Research Inst for Sport and Exercise, University of Canberra, Canberra, ACT, Australia. Jeacocke is with the Australian Institute of Sport, Canberra, ACT, Australia. Mesquida is with the Dept of Applied Science, Technological University Dublin-Tallaght Campus, Dublin, Ireland. Etxebarria (naroa.etxebarria@canberra.edu.au) is corresponding author.

\section{Pacing Strategy During the 10-km Run}

Athletes from different single-discipline endurance sports often make comments such as "I let her/him go, I knew I would catch her/him if I focused on my race plan and I didn't let her/him affect what I came here to do, which is to execute my race as well as I could" or "her/his finish is very strong so I had to push the pace at the start, make her/him uncomfortable and not let her/him execute their perfect race." Such statements demonstrate the importance of an athlete knowing their strengths and weaknesses and those of their competitors, and not letting race dynamics or other competitors negatively influence the race performance. Most such comments relate to pacing, where energy use needs to be distributed effectively to utilize all available resources while limiting premature fatigue in order to complete the race in the fastest possible time,$^{8}$ which is particularly crucial in long-distance races such as triathlon. In endurance races, careful and continuous management of effort and energy expenditure is necessary to maintain a high speed throughout all 3 disciplines. ${ }^{7}$

It appears that adoption of a negative pacing strategy (ie, where speed gradually increases) can improve endurance performance. ${ }^{9}$ Indeed, during a simulated triathlon, performing the first $1 \mathrm{~km}$ of a $10-\mathrm{km}$ run $5 \%$ slower than the overall mean speed resulted in a faster $10-\mathrm{km}$ run. ${ }^{6}$ The benefits of a negative pacing strategy likely include a reduced rate of carbohydrate depletion, lower excessive oxygen consumption $\left(\mathrm{VO}_{2}\right)$, and/or limiting accumulation of fatigue-related metabolites. ${ }^{9}$ However, despite the evidence favoring a negative pace strategy, single race-based evidence, somewhat counterintuitively, indicates that triathletes adopt a positive pacing strategy through the run section. ${ }^{4}$ Examining multiple race performances of a large cohort of top competitors during elite triathlon races would confirm how athletes race the final $10-\mathrm{km}$ run, and whether there is scope for improving pacing strategies that could enhance overall race performance.

We took the opportunity to examine the performances of a total of 171 different elite male triathletes (age 27 [4] y) over 726 race outcomes over the $10-\mathrm{km}$ run section of 14 elite Olympic distance triathlon races (International Triathlon Union Triathlon 
World Cup and World Triathlon Series events) over 3 consecutive years. The selected races included in this analysis had 52 (6) (mean [SD]) participants in each. The elapsed time for both the entire run section and each of four $\sim 2.5-\mathrm{km}$ laps were obtained from the International Triathlon Union on-course timing system. Laps in elite triathlon races can often vary slightly in distance given variations in racing lines, transition zones, and the finish chute. Therefore, we measured each run course using a trundle wheel, taking the "racing line" and reported distance to the nearest meter. These measurements were then used to calculate pacing times, in the form of mean running pace per kilometer (in seconds) over each of four $\sim 2.5-\mathrm{km}$ laps. Mean running pace (in minutes:seconds per kilometer) was calculated for each $\sim 2.5-\mathrm{km}$ lap and $10-\mathrm{km}$ run section. We then correlated each of the individual run laps with both total $10-\mathrm{km}$ run time and overall race time to establish the patterns of pacing.

A positive pacing strategy, where an athlete's speed decreases over the duration of the race, was utilized by almost all triathletes in these International Triathlon Union races (Figure 1). The first of the four $\sim 2.5-\mathrm{km}$ laps was substantially faster compared with the rest, with $6.9, \pm 0.2 \% ; 8.8, \pm 0.3 \%$; and $12.0, \pm 0.5 \%$ (standardized difference, $\pm 90 \%$ confidence limits [CL]) slower times for laps 2, 3, and 4 (compared with lap 1). The last lap was substantially slower than laps 2 and 3 . These results clearly indicate a positive pacing profile in contemporary elite triathlon in contrast to the evidence in many other sports (and research studies) that an even-paced or negative profile (with a fast finishing endspurt) is preferred. Moreover, the mean pace in the first lap had the lowest correlation ( $r=.82, \pm .02$; correlation, $\pm 90 \% \mathrm{CL}$ ) with the $10-\mathrm{km}$ total run time (Table 1$)$, with the second $(r=.93, \pm .01)$ and third $(r=.94, \pm .01)$ laps showing a substantially higher correlation (difference $r \geq .10$ ). The overall race performance outcome was best associated with the run performance $(r=.82, \pm .02$; correlation, $\pm 90 \% \mathrm{CL})$ compared with the swim $(r=.35, \pm .05)$ and cycle $(r=.43, \pm .05)$ sections. The top runners implemented a less variable run pace throughout the $10 \mathrm{~km}(\sim 4 \%)$ than the rest of the field $(\sim 5 \%)$, which was moderately correlated with the overall $10-\mathrm{km}$ run time $(r=.40, \pm .06)$.

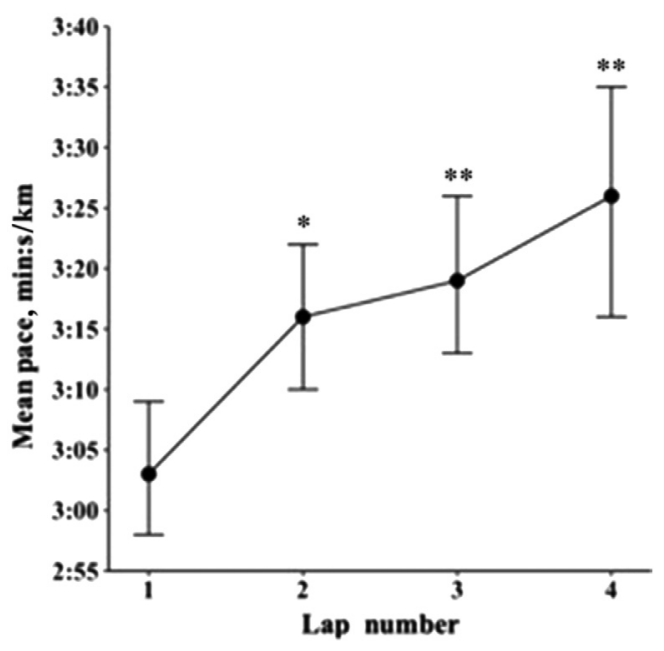

Figure 1 - Mean running pace (in minutes:seconds per kilometer) for male participants in International Triathlon Union World Triathlon Series races for each of the four $\sim 2.5-\mathrm{km}$ laps in the $10-\mathrm{km}$ run section. Data are shown as mean (SD). Standardized changes compared with lap 1 are represented as *"large" and **“very large."

\section{Race Performance Optimizing Strategies}

The question is how to reconcile this recurring issue of a positive pacing strategy evident in Olympic distance triathlon races, in contradiction to the conventional (research-based) notion that a negative or even-paced strategy is preferred. These outcomes over 14 elite Olympic distance triathlon races support previous single race-based research showing triathletes typically adopt a positive pacing strategy where speed gradually declines, but not a final increase in pace ("J" shaped pacing) described in these races prior to $2012-2013 .{ }^{10}$ A positive pacing strategy is likely linked to a higher metabolic demand and greater accumulation of fatiguerelated metabolites (blood lactate) compared with a negative pace strategy. ${ }^{6}$ Uncompensable pace at the start of a $10-\mathrm{km}$ run could result in early accumulation of metabolites (ie, blood lactate), which impair overall performance. This strategy will see the athlete decrease their running pace gradually eliciting a suboptimal run performance and jeopardizing the overall race outcome. Of course, tactics and race dynamics during the preceding swim and bike sections will also affect the pace athletes self-select in the initial stages of the run. However, despite the psychological optimism some athletes might experience from a fast start, and positioning themselves ahead of other competitors early on, the final run result might be hindered if they cannot sustain that pace. Therefore, as $>80 \%$ of the overall race outcome is explained by the $10-\mathrm{km}$ run performance, athletes could benefit from adopting a more evenpaced strategy that allows for the fastest overall run time. This proposition awaits further support after additional trialing and rehearsal in the field by triathletes.

In our analyses, we established that the top 8 best run times of each race were achieved by adopting a more even-paced run strategy than the rest of the field over the course of the $10 \mathrm{~km}$. Less variability in running pace is associated with better run performances during triathlon ${ }^{7}$ and also during 10-km races without any preceding swimming or cycling. ${ }^{11}$ Race tactics and dynamics, placing during the swim and the cycle sections, transitions, and other competitors' race strategies need to be considered in triathlon. However, the large association of the run time to overall race placing warrants a race strategy that prioritizes and enhances the final $10-\mathrm{km}$ run time. Perhaps individual pacing profiles ${ }^{12}$ that align with physiological attributes of each athlete should be considered, accounting for how physiological responses during the run section are affected by preceding cycling. ${ }^{13}$ For example, a triathlete with a large anaerobic energy reserve could benefit from adopting a fast start or employing a mid-race surge to break away from the competition, as anaerobic power can vary substantially between triathletes. ${ }^{14}$ Conversely, a triathlete capable of running at a relatively high speed while obtaining much of the necessary energy from the aerobic system, and hence sparing the anaerobic energy reserve, could benefit from a more even-paced approach. Individual race-pace enhancement where running pace strategies are matched to physiological profiles of each athlete should promote the best run and overall performances.

The high variability in race conditions both in regard to swimming (swimming in open water or lake and the use [or not] of a swimsuit) and also cycling courses (technicality, topography, and race dynamics) in Olympic distance triathlon makes it difficult to isolate and control all factors that influence triathlon performance. However, there are aspects of triathlon racing that triathletes should try to control and influence so they can maximize their strengths as an athlete (fast running ability) and minimize exposure to the less strong skills and abilities (eg, poorer technical 
Table 1 Correlation Matrix Between Mean Running Pace in Each 2.5-km Lap and Overall 10-km Run Time for Each of the 14 International Triathlon Union World Triathlon Series Races

\begin{tabular}{|c|c|c|c|c|c|}
\hline Race & No. of participants & Lap 1 & Lap 2 & Lap 3 & Lap 4 \\
\hline Chicago ${ }^{\text {Year I }}$ & 61 & .84 & .89 & .92 & .89 \\
\hline Rio & 60 & .82 & $.94^{\mathrm{a}}$ & .90 & .93 \\
\hline Yokohama & 50 & .89 & .97 & .96 & .94 \\
\hline Cape Town Year I & 55 & .80 & $.93^{\mathrm{a}}$ & $.97^{\mathrm{a}}$ & .92 \\
\hline Gold Coast & 51 & .85 & .94 & .96 & .95 \\
\hline Auckland ${ }^{\text {YearI }}$ & 48 & .88 & .95 & .94 & .86 \\
\hline Edmonton & 58 & .90 & .98 & .97 & .93 \\
\hline Chicago Year II & 52 & .80 & $.95^{\mathrm{a}}$ & $.97^{\mathrm{a}}$ & .92 \\
\hline Yokohama & 48 & .93 & .92 & .94 & .93 \\
\hline Cape Town ${ }^{\text {Year II }}$ & 49 & .92 & .93 & .91 & .95 \\
\hline Auckland $^{\text {Year II }}$ & 44 & .82 & .93 & $.94^{\mathrm{a}}$ & .88 \\
\hline London & 60 & .84 & $.98^{\mathrm{a}}$ & $.96^{\mathrm{a}}$ & No data \\
\hline Stockholm & 43 & .78 & .85 & $.96^{\mathrm{a}}$ & $.91^{\mathrm{a}}$ \\
\hline Madrid & 47 & .91 & .95 & .95 & .95 \\
\hline Overall & 726 & .82 & $.93^{\mathrm{a}}$ & $.94^{\mathrm{a}}$ & .88 \\
\hline $\pm 90 \% \mathrm{CL}$ & & .02 & .01 & .01 & .01 \\
\hline
\end{tabular}

${ }^{\mathrm{a}}$ Substantial difference $(r>.10)$ in correlation between lap pace and 10-km run time compared with lap 1.

cycling skills). The order in which athletes come out of both the swimming and cycling sections results in critical transition points where race dynamics take shape. Strong swimmers will come out of the water first and try to create a gap between themselves and any stronger runners who might have been left behind during the swim. Bunch riding during the cycle is beneficial to the energy savings that drafting provides, but also provides the opportunity to create/ increase a gap between a leading group composed of strong cyclists and weaker ones who might be stronger runners. This strategy should assist the stronger cyclists who might be less performed runners obtain a modest head start for the last $10-\mathrm{km}$ run.

Triathletes, as part of their training and competition readiness plan, ${ }^{15}$ should consider and implement a race strategy that is likely to yield a competitive advantage. Race dynamics during the initial swim and cycle sections should play to the strengths of slower runners, whereas fast runners should maximize drafting by seeking shelter behind other competitors during the swim and cycle sections. For example, it would make little competitive sense for a slow runner who is a strong cyclist to sit at the back of the cycling bunch during the cycle section without pushing the pace. It would be better for this athlete to hurt faster runners who are weaker cyclists and create a substantial gap ahead of the final 10-km run. Similarly, it would be risky, physiologically, to start the $10-\mathrm{km}$ run at a pace that is substantially higher than the average pace the athlete is able to sustain for $\sim 30$ minutes. Developing cycling technical skills that allow triathletes to maintain momentum during technical courses, and minimize constant deceleration and acceleration, would prove beneficial for strong cyclists who break away or weaker cyclists who "save their legs" as much as possible for the subsequent run. Finally, a stronger focus on individual performance is suggested, rather than pacing being dictated or detrimentally affected by other competitors' pace and/or race dynamics. Individual pacing profiles that align with each athlete's physiological attributes and skills should enhance triathletes' performance.

\section{Summary}

Triathletes typically adopt a positive pacing strategy during the final run section of the elite International Triathlon Union World Cup and World Championship series. However, this strategy is at odds with evidence pointing to the benefits of a negative pacing strategy during endurance events. Given the small margins in performance between world-class triathletes, small differences in pacing strategy, especially minimizing variations in pacing, are worthwhile avenues for triathletes and coaches to enhance performance. Trying to keep up with a leading group while forsaking the athlete's individual race plan might compromise $10-\mathrm{km}$ run performance, overall race outcome, and loss of valuable ranking points. Triathletes and coaches should consider adopting a more conservative pacing strategy in the first lap of the 10-km run section to perform better in subsequent laps and enhance overall race performance.

\section{Acknowledgments}

The lead author thanks Mark Pearce, and all the incredibly talented athletes and ingenious coaches whose insights on triathlon training, race preparation, and racing strategies have inspired and helped inform this commentary. The authors also acknowledge the cooperation of Triathlon Australia and Dr Annette Eastwood for their contributions to the data collection.

\section{References}

1. Hausswirth C, Brisswalter J. Strategies for improving performance in long duration events: Olympic distance triathlon. Sports Med. 2008; 38(11):881-891. PubMed ID: 18937520 doi:10.2165/00007256200838110-00001

2. Ofoghi B, Zeleznikow J, Macmahon C, Rehula J, Dwyer DB. Performance analysis and prediction in triathlon. J Sports Sci. 2016; 34(7):607-612. PubMed ID: 26177783 doi:10.1080/02640414.2015. 1065341 
3. Vleck VE, Bentley DJ, Millet GP, Bürgi A. Pacing during an elite Olympic distance triathlon: comparison between male and female competitors. J Sci Med Sport. 2008;11(4):424-432. PubMed ID: 17350889 doi:10.1016/j.jsams.2007.01.006

4. Le Meur Y, Hausswirth C, Dorel S, et al. Influence of gender on pacing adopted by elite triathletes during a competition. Eur J Appl Physiol. 2009;106(4):535-545. PubMed ID: 19340453 doi:10.1007/ s00421-009-1043-4

5. Etxebarria N, D'Auria S, Anson JM, Pyne DB, Ferguson RA. Variability in power output during cycling in international Olympicdistance triathlon. Int J Sports Physiol Perform. 2014;9(4):732-734. PubMed ID: 24235776 doi:10.1123/ijspp.2013-0303

6. Hausswirth C, Le Meur Y, Bieuzen F, Brisswalter J, Bernard T. Pacing strategy during the initial phase of the run in triathlon: influence on overall performance. Eur J Appl Physiol. 2010;108(6): 1115-1123. PubMed ID: 20024576 doi:10.1007/s00421-009-1322-0

7. Le Meur Y, Bernard T, Dorel S, et al. Relationships between triathlon performance and pacing strategy during the run in an international competition. Int J Sports Physiol Perform. 2011;6(2):183-194. PubMed ID: 21725104 doi:10.1123/ijspp.6.2.183

8. Foster C, De Koning JJ, Hettinga F, et al. Pattern of energy expenditure during simulated competition. Med Sci Sports Exerc. 2003;35(5):826831. PubMed ID: 12750593 doi:10.1249/01.MSS.0000065001.17658.68

9. Abbiss CR, Laursen PB. Describing and understanding pacing strategies during athletic competition. Sports Med. 2008;38(3):
239-252. PubMed ID: 18278984 doi:10.2165/00007256-20083803000004

10. Wu SS, Peiffer JJ, Brisswalter J, Nosaka K, Abbiss CR. Factors influencing pacing in triathlon. Open Access J Sports Med. 2014;5:223-234. PubMed ID: 25258562 doi:10.2147/OAJSM. S44392

11. Tucker R, Lambert MI, Noakes TD. An analysis of pacing strategies during men's world-record performances in track athletics. Int $J$ Sports Physiol Perform. 2006;1(3):233-245. PubMed ID: 19116437 doi:10.1123/ijspp.1.3.233

12. Lima-Silva AE, Bertuzzi RC, Pires FO, et al. Effect of performance level on pacing strategy during a 10-km running race. Eur J Appl Physiol. 2010;108(5):1045-1053. PubMed ID: 20012450 doi:10. 1007/s00421-009-1300-6

13. Etxebarria N, Hunt J, Ingham S, Ferguson R. Physiological assessment of isolated running does not directly replicate running capacity after triathlon-specific cycling. J Sports Sci. 2014;32(3):229-238. PubMed ID: 24016097 doi:10.1080/02640414.2013.819520

14. Bernard T, Hausswirth C, Le Meur Y, et al. Distribution of power output during the cycling stage of a Triathlon World Cup. Med Sci Sports Exerc. 2009;41(6):1296-1302. PubMed ID: 19461535 doi: 10.1249/MSS.0b013e318195a233

15. Etxebarria N, Mujika I, Pyne DB. Training and competition readiness in triathlon. Sports. 2019;7(5):101. PubMed ID: 31035719 doi:10. 3390/sports 7050101 